\title{
EXISTENCE OF ALMOST SPLIT SEQUENCES VIA REGULAR SEQUENCES
}

\author{
HOSSEIN ESHRAGHI
}

(Received 5 August 2012; accepted 28 December 2012; first published online 18 March 2013)

\begin{abstract}
Let $(R, \mathrm{~m})$ be a Cohen-Macaulay complete local ring. We will apply an inductive argument to show that for every nonprojective locally projective maximal Cohen-Macaulay object $\mathcal{X}$ of the morphism category of $R$ with local endomorphism ring, there exists an almost split sequence ending in $X$. Regular sequences are exploited to reduce the Krull dimension of $R$ on which the inductive argument is established. Moreover, the Auslander-Reiten translate of certain objects is described.
\end{abstract}

2010 Mathematics subject classification: primary 16G70; secondary $16 \mathrm{G} 50$.

Keywords and phrases: almost split sequence, Cohen-Macaulay modules, morphism category.

\section{Introduction and preliminaries}

1.1. Let $R$ be a ring. Recall that an $R$-homomorphism $g: B \rightarrow C$ is said to be right almost split provided it is not a split epimorphism and factors every $R$-homomorphism $X \rightarrow C$ which is not a split epimorphism. A nonsplit exact sequence $0 \rightarrow A \stackrel{f}{\rightarrow} B \stackrel{g}{\rightarrow}$ $C \rightarrow 0$ of $R$-modules is called an almost split sequence provided the endomorphism ring of $A$ is local and the $R$-homomorphism $g$ is right almost split.

The notion of almost split sequences was first defined by Auslander and Reiten [3] in their study of the module category of an Artin algebra. It was in this setting that they gave the first existence theorem for almost split sequences. Later, almost split sequences were studied in various contexts such as orders over Gorenstein rings [2] and the category of maximal Cohen-Macaulay modules over a Henselian CohenMacaulay local ring which admits a canonical module; see [13, 17].

For an associative ring $R$ with unity, denote by $\operatorname{Mod} R(\operatorname{respectively,} \bmod R$ ) the category of all (respectively, finitely generated) $R$-modules and by $\mathcal{H}(R)$ the morphism category of $R$ whose objects are all $R$-homomorphisms $X \stackrel{f}{\rightarrow} Y$ in $\operatorname{Mod} R$ and whose morphisms are given by commutative squares. The subcategory of $\mathcal{H}(R)$ consisting of finitely generated objects will be denoted by $\mathcal{H}_{f g}(R)$. In this note we will discuss the existence of almost split sequences in the morphism category of a

(C) 2013 Australian Mathematical Publishing Association Inc. 0004-9727/2013 \$16.00 
commutative ring $R$. It will be proved that for certain $R$, any indecomposable locally projective maximal Cohen-Macaulay object of $\mathcal{H}(R)$, in the sense we will define later, is the end term of an almost split sequence. We prove this result by using an inductive argument on the Krull dimension $d$ of $R$. Actually, the induction is done by using regular sequences to reduce the dimension of the base ring. We very much hope that this inductive procedure may be improved and generalised to other contexts.

Here we give a preview of the paper and state the main theorems. The rest of this section is devoted to providing some preliminaries. In its last part we study the notion of tensor product in the category $\mathcal{H}(R)$ by use of which we will prove the isomorphism of Theorem 1.8. We remark that this isomorphism has been proved in [4] in the case of commutative Gorenstein rings and also in [17] by applying spectral sequences. We will follow the proof given in [13]. This is fruitful from the point of view that it enables us to understand the explicit description of the Auslander-Reiten translate $\tau_{\mathcal{H}}$, as will be introduced later on, of some certain objects of $\mathcal{H}(R)$.

In Section 2 our main tool is the following theorem in which $\mathfrak{C}(\mathcal{H})$ is the subcategory of $\mathcal{H}_{f g}(R)$ consisting of all objects which are maximal Cohen-Macaulay when considered as $R$-modules.

Theorem A. Let $(R, \mathrm{~m})$ be a complete Cohen-Macaulay local ring of dimension $d$ and let $\mathcal{X}$ be a nonprojective locally projective object of $\mathfrak{S}(\mathcal{H})$ with local endomorphism ring. Assume that $x \in \mathfrak{m}$ is an $R$ - and $\mathcal{X}$-sequence (see Definition 2.2). Then for some natural number $n$, there exists a nonzero natural map

$$
\eta_{\mathcal{X}}: \operatorname{Ext}_{\mathcal{H}\left(R / x^{n} R\right)}^{1}\left(\mathcal{X} / x^{n} \mathcal{X}, \tau_{\mathcal{H}\left(R / x^{n} R\right)}\left(\mathcal{X} / x^{n} \mathcal{X}\right)\right) \longrightarrow \operatorname{Ext}_{\mathcal{H}(R)}^{1}\left(\mathcal{X}, \tau_{\mathcal{H}(R)}(\mathcal{X})\right)
$$

which has the property that every socle element of the right-hand side is the image of a socle element of the left-hand side.

Proof. See Theorem 2.8.

Then an inductive procedure will be given to prove the following theorem.

Theorem B. Let $R$ be as in Theorem A. For any nonprojective locally projective maximal Cohen-Macaulay object (see Definition 2.1) $X=X \stackrel{f}{\rightarrow} Y$ of $\mathcal{H}(R)$ with local endomorphism ring, there exists an almost split sequence

$$
0 \longrightarrow \tau_{\mathcal{H}}(X) \longrightarrow \mathcal{E} \longrightarrow X \longrightarrow 0
$$

in $\mathfrak{C}(\mathcal{H})$.

Also, in an effort to recognise the object $\tau_{\mathcal{H}}(\mathcal{X})$, we prove the following result.

THeOREM C. With the hypothesis as above, let $\omega$ be the canonical module of $R$. If $\operatorname{Ext}_{R}^{1}(\operatorname{Coker}(f), R)=0$, then $\tau_{\mathcal{H}}(\mathcal{X})$ is a representative of the induced map $\operatorname{Hom}_{R}\left(\operatorname{syz}^{d} \operatorname{Tr} Y, \omega\right) \longrightarrow \operatorname{Hom}_{R}\left(\operatorname{syz}^{d} \operatorname{Tr} \operatorname{Coker}(f), \omega\right)$. 
We remark that there also exist dual statements concerning indecomposable noninjective objects and the inverse Auslander-Reiten translation which can be proved using the same inductive method; so we do not give them.

1.2. It is known that for a ring $R$, the projective objects of $\mathcal{H}(R)$ are direct sums of elements of the form $P \stackrel{1}{\rightarrow} P$ and $0 \rightarrow P$ where $P$ runs through all projective $R$-modules. Dually, every injective element in $\mathcal{H}(R)$ is a product of elements of the form $I \stackrel{1}{\rightarrow} I$ and $I \rightarrow 0$ where $I$ runs through all injective $R$-modules. Actually, the category $\mathcal{H}(R)$ is clearly equivalent to the category of modules over the path algebra $R Q$ of the quiver $Q: \bullet \rightarrow \bullet$ over $R$; see [5]. Hence, among other sources, the latter two statements about projective and injective elements in $\mathcal{H}(R)$ are just special cases of the main results in [8,9] respectively. Note that if $R$ is commutative noetherian then $\mathcal{H}(R)$ may be considered as a noetherian $R$-algebra in the sense of [2].

1.3. It is a standard result that every finitely generated object $X \stackrel{f}{\rightarrow} Y$ of $\mathcal{H}(R)$ admits a projective cover when $R$ is a local ring. So we just recall the structure of the projective covers. Let $P \stackrel{\varphi}{\rightarrow} X$ and $Q \stackrel{\psi}{\rightarrow} \operatorname{Coker}(f)$ be projective covers of the $R$-modules $X$ and $\operatorname{Coker}(f)$, respectively. If $\pi_{f}: Y \rightarrow \operatorname{Coker}(f)$ is the natural map, then there exists a homomorphism $\gamma: Q \rightarrow Y$ with $\pi_{f} \gamma=\psi$. This gives rise to a map $(\varphi, f \varphi \oplus \gamma):(P \stackrel{i}{\rightarrow} P \oplus Q) \longrightarrow(X \stackrel{f}{\rightarrow} Y)$, where $i$ is the natural injection. Then we have the following lemma.

Lemma 1.1. Let $X \stackrel{f}{\rightarrow} Y$ belong to $\mathcal{H}_{f g}(R)$. With the above notation, $(\varphi, f \varphi \oplus \gamma):(P \stackrel{i}{\rightarrow}$ $P \oplus Q) \longrightarrow(X \stackrel{f}{\rightarrow} Y)$ is a projective cover.

1.4. Recall that, for a ring $R$, the functor $(-)^{*}=\operatorname{Hom}_{R}(-, R): \operatorname{Mod} R \longrightarrow \operatorname{Mod} R^{\text {op }}$ induces a duality between the full subcategories of finitely generated projective modules over $R$ and those over $R^{\text {op }}$. Now let $X \stackrel{f}{\rightarrow} Y$ belong to $\mathcal{H}(R)$ and let $\pi_{f}$ be the natural map $Y \rightarrow \operatorname{Coker}(f)$. The object $\operatorname{Coker}(f)^{*} \stackrel{\pi_{f}^{*}}{\rightarrow} Y^{*}$ of $\mathcal{H}\left(R^{\text {op }}\right)$ will be denoted by $(X \stackrel{f}{\rightarrow} Y)^{*}$ and if $g=\left(g_{1}, g_{2}\right):(X \stackrel{f}{\rightarrow} Y) \longrightarrow\left(X^{\prime} \stackrel{f^{\prime}}{\rightarrow} Y^{\prime}\right)$ is a morphism in $\mathcal{H}(R)$, then $g^{*}:\left(X^{\prime} \stackrel{f^{\prime}}{\rightarrow} Y^{\prime}\right)^{*} \longrightarrow(X \stackrel{f}{\rightarrow} Y)^{*}$ is just the obvious map induced by the homomorphisms $g_{1}^{*}$ and $g_{2}^{*}$. This turns out to be an appropriate extension of the $R$-dual functor mentioned above to the morphism category of $R$, which will again be denoted by $(-)^{*}$. Evidently, $(-)^{*}$ induces a duality between the subcategory of projective objects in $\mathcal{H}_{f g}(R)$ and those in $\mathcal{H}_{f g}\left(R^{\mathrm{op}}\right)$.

Recall that if $\mathcal{P}_{1} \stackrel{\psi_{0}}{\longrightarrow} \mathcal{P}_{0} \stackrel{\psi}{\longrightarrow} \mathcal{X} \longrightarrow 0$ is a projective presentation of a finitely generated object $\mathcal{X}=X \stackrel{f}{\rightarrow} Y$ in $\mathcal{H}(R)$ then its Auslander-Bridger transpose $\operatorname{Tr}_{\mathcal{H}}(\mathcal{X})$, or simply $\operatorname{Tr}(\mathcal{X})$, is defined as $\operatorname{Coker}\left(\mathcal{P}_{0}^{*} \stackrel{\psi_{0}^{*}}{\longrightarrow} \mathcal{P}_{1}^{*}\right)$. Hence $\operatorname{Tr}{ }_{\mathcal{H}}(\mathcal{X})$ depends on the choice of the projective presentation of $\mathcal{X}$. However, it can be seen that if $Q_{1} \stackrel{\varphi_{0}}{\longrightarrow} Q_{0} \stackrel{\varphi}{\longrightarrow} \mathcal{X} \longrightarrow 0$ is another presentation by projective objects, then 
$\operatorname{Coker}\left(Q_{0}^{*} \stackrel{\varphi_{0}^{*}}{\longrightarrow} Q_{1}^{*}\right)$ and $\operatorname{Coker}\left(\mathcal{P}_{0}^{*} \stackrel{\psi_{0}^{*}}{\longrightarrow} \mathcal{P}_{1}^{*}\right)$ are isomorphic up to projective direct summands. Now, for any two objects $\mathcal{X}$ and $\mathcal{Y}$ of $\mathcal{H}(R)$, let $\mathfrak{P}(\mathcal{X}, \mathcal{Y})$ consist of those maps from $\mathcal{X}$ to $\mathcal{Y}$ in $\mathcal{H}(R)$ which factor through a projective object. Also, let the stable category $\underline{\mathcal{H}}(R)$ have the objects of $\mathcal{H}(R)$ as its objects and $\operatorname{Hom}_{\mathcal{H}(R)}(X, Y)=$ $\operatorname{Hom}_{\mathcal{H}(R)}(\mathcal{X}, \mathcal{Y}) / \mathfrak{P}(\mathcal{X}, \mathcal{Y})$ for any $\mathcal{X}$ and $\mathcal{Y}$. Then, even though there is not a functor $\operatorname{Tr}: \mathcal{H}_{f g}(R) \longrightarrow \mathcal{H}_{f g}\left(R^{\mathrm{op}}\right)$, there exists a functor $\operatorname{Tr}: \underline{\mathcal{H}}_{f g}(R) \longrightarrow \underline{\mathcal{H}}_{f g}\left(R^{\mathrm{op}}\right)$ which is easily seen to be a duality. It is also straightforward to see that for any two objects $\mathcal{X}$ and $\mathcal{Y}$ of $\mathcal{H}_{f g}(R)$, there exists an isomorphism $\underline{\operatorname{Hom}}_{\mathcal{H}(R)}(\mathcal{X}, \mathcal{Y}) \simeq \underline{\operatorname{Hom}}_{\mathcal{H}\left(R^{\text {op }}\right)}$ $(\operatorname{Tr}(\mathcal{X}), \operatorname{Tr}(\mathcal{Y}))$.

We emphasise that when $R$ is local, then according to Lemma 1.1 we may consider minimal projective presentations to define and compute the transpose.

In the following proposition we intend to obtain the description of the transpose of the objects in $\mathcal{H}_{f g}(R)$. Here, the transpose in $\bmod R$ of a finitely generated $R$-module $M$ is denoted by $\operatorname{Tr}(M)$.

Proposition 1.2. Let $R$ be a commutative local ring and $\mathcal{X}=X \stackrel{f}{\rightarrow} Y$ belong to $\mathcal{H}_{f g}(R)$ with $f$ a monomorphism. Then $\operatorname{Tr}_{\mathcal{H}}(X \stackrel{f}{\rightarrow} Y)$ is a representative of the induced map $\operatorname{Tr}(\operatorname{Coker}(f)) \stackrel{\operatorname{Tr}\left(\pi_{f}\right)}{\longrightarrow} \operatorname{Tr}(Y)$ as an object in $\mathcal{H}_{f g}(R)$. If, moreover, $\operatorname{Ext}_{R}^{1}(\operatorname{Coker}(f), R)=0$, then the sequence

$$
0 \longrightarrow \operatorname{Tr}(\operatorname{Coker}(f)) \stackrel{\operatorname{Tr}\left(\pi_{f}\right)}{\longrightarrow} \operatorname{Tr}(Y) \stackrel{\operatorname{Tr}(f)}{\longrightarrow} \operatorname{Tr}(X) \longrightarrow 0
$$

of R-modules is exact.

Proof. Assume that $P_{1} \stackrel{\xi_{0}}{\longrightarrow} P_{0} \stackrel{\xi}{\longrightarrow} X \longrightarrow 0$ is a minimal projective presentation of $X$ and choose projective $R$-modules $Q_{0}$ and $Q_{1}$ such that, by Lemma 1.1,

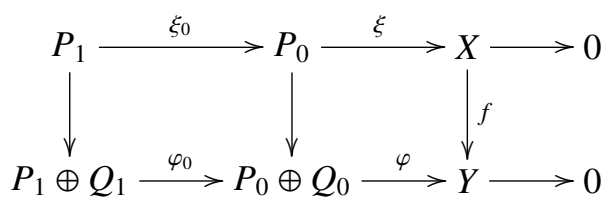

is a minimal projective presentation of $X \stackrel{f}{\rightarrow} Y$ in $\mathcal{H}_{f g}(R)$. In view of the definition of the transpose of an object given above, one may apply the functor $(-)^{*}$ to obtain the 
commutative exact diagram

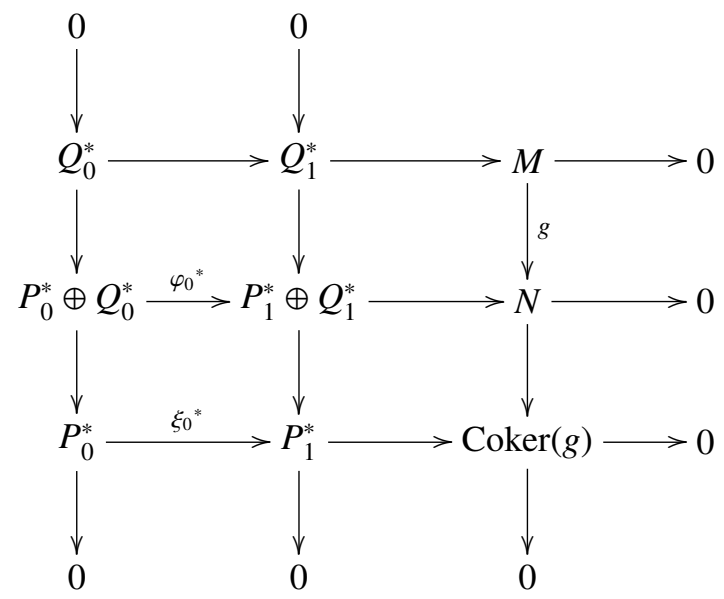

where the lower row is obtained by computing the cokernels of the vertical maps, all the maps are just the obvious ones, and $M \stackrel{g}{\rightarrow} N$ is the transpose of $X \stackrel{f}{\rightarrow} Y$. Since $P_{1} \stackrel{\xi_{0}}{\longrightarrow} P_{0} \stackrel{\xi}{\longrightarrow} X \longrightarrow 0$ is a minimal projective presentation of $X$, we get that $\operatorname{Coker}(g) \simeq \operatorname{Tr}(X)$. On the other hand, since $f$ is mono, the Snake lemma implies that $Q_{1} \rightarrow Q_{0} \rightarrow \operatorname{Coker}(f) \rightarrow 0$ is a minimal projective presentation, that is, $M \simeq$ $\operatorname{Tr}(\operatorname{Coker}(f))$. Also, again since $f$ is a monomorphism, [11, Lemma 2.2] gives that $P_{1} \oplus Q_{1} \stackrel{\varphi_{0}}{\longrightarrow} P_{0} \oplus Q_{0} \stackrel{\varphi}{\longrightarrow} Y \longrightarrow 0$ is actually a minimal projective presentation of $Y$ and consequently $N \simeq \operatorname{Tr}(Y)$. Finally, if $\operatorname{Ext}_{R}^{1}(\operatorname{Coker}(f), R)=0$ then add the exact sequence $0 \rightarrow \operatorname{Coker}(f)^{*} \stackrel{\pi_{f}^{*}}{\longrightarrow} Y^{*} \stackrel{f^{*}}{\longrightarrow} X^{*} \longrightarrow 0$ as the left column to the above diagram and deduce then that $g$ is a monomorphism.

REMARK 1.3. We point out that, by the results in [9], every Gorenstein projective object in $\mathcal{H}(R)$ satisfies the hypothesis of the above proposition. So also does every object $X \stackrel{f}{\rightarrow} Y$ of $\mathcal{H}(R)$ with $f$ a monomorphism and Coker $(f)$ maximal Cohen-Macaulay in the usual sense, when $R$ is a local Gorenstein ring.

1.5. In this part we aim to settle the following Theorem 1.8. This has already been established in some settings such as algebras over commutative Gorenstein rings [4]. We just provide prerequisites and follow the argument given in [13] for the case of local Cohen-Macaulay rings admitting a canonical module. So let $R$ be a commutative ring and $\mathfrak{C}(\mathcal{H})$ be the subcategory of $\mathcal{H}_{f g}(R)$ whose objects are maximal CohenMacaulay as $R$-modules. Our reference for Cohen-Macaulay rings and modules and the canonical module of a ring is [6].

Firstly, we need to recognise explicitly the notion of tensor product in the category $\mathcal{H}(R)$. So let $\mathcal{X}=X \stackrel{f}{\rightarrow} Y$ belong to $\mathcal{H}(R)$. Define a functor $\operatorname{Hom}_{R}(X,-): \operatorname{Mod} R \longrightarrow$ $\mathcal{H}(R)$ in the following way: for any $R$-module $U, \operatorname{Hom}_{R}(X, U)$ is the element 
$\operatorname{Hom}_{R}(Y, U) \stackrel{\operatorname{Hom}_{R}(f, U)}{\longrightarrow} \operatorname{Hom}_{R}(X, U)$ and, if $U \stackrel{g}{\rightarrow} V$ is a homomorphism of $R$-modules, we let $\operatorname{Hom}_{R}(X, g)$ act in the obvious way. Then it is easy to verify that $\operatorname{Hom}_{R}(X,-)$ is indeed a left exact functor which, by the elementary properties of the usual $\operatorname{Hom}_{R}$ functor, is product preserving. Hence by [15, Corollary 3.39] it has a left adjoint. So now let $-\otimes_{\mathcal{H}} \mathcal{X}: \mathcal{H}(R) \longrightarrow \operatorname{Mod} R$ be the left adjoint of the functor $\operatorname{Hom}_{R}(\mathcal{X},-)$. Therefore, for any $R$-module $U$ and any object $\mathcal{M}$ of $\mathcal{H}(R)$, there exists an isomorphism

$$
\operatorname{Hom}_{R}\left(\mathcal{M} \otimes_{\mathcal{H}} \mathcal{X}, U\right) \simeq \operatorname{Hom}_{\mathcal{H}}\left(\mathcal{M}, \operatorname{Hom}_{R}(X, U)\right)
$$

which is called the Hom $-\otimes$ adjoint isomorphism.

To see that $-\otimes_{\mathcal{H}} \mathcal{X}$ has all expected attributes as a tensor product functor, we refer to [16] where this approach has been applied to the more general setting of complexes of modules over commutative rings; the arguments there permeate to $\mathcal{H}(R)$. For instance, it can easily be seen that $-\otimes_{\mathcal{H}} \mathcal{X}$ preserves direct limits. Moreover, if $\mathcal{P}$ is any projective object in $\mathcal{H}(R)$, then $-\otimes_{\mathcal{H}} \mathcal{P}$ is an exact functor. Note that for arbitrary $\mathcal{X}$, $-\otimes_{\mathcal{H}} \mathcal{X}$ is right exact, being the left part of an adjoint pair.

However, we will show below that this behaviour should not be thought of as far reaching.

Lemma 1.4. For any $\mathcal{X}=X \stackrel{f}{\rightarrow} Y$ in $\mathcal{H}(R)$, there are $R$-isomorphisms $(0 \rightarrow R) \otimes_{\mathcal{H}} \mathcal{X} \simeq$ $X$ and $(R \rightarrow R) \otimes_{\mathcal{H}} X \simeq Y$.

Proof. Let $U$ be any $R$-module. From the Hom $-\otimes$ adjoint isomorphism,

$$
\begin{aligned}
\operatorname{Hom}_{R}\left((0 \rightarrow R) \otimes_{\mathcal{H}} \mathcal{X}, U\right) & \simeq \operatorname{Hom}_{\mathcal{H}}\left((0 \rightarrow R), \operatorname{Hom}_{R}(X, U)\right) \\
& \left.\simeq \operatorname{Hom}_{\mathcal{H}}\left((0 \rightarrow R), \operatorname{Hom}_{R}(Y, U) \rightarrow \operatorname{Hom}_{R}(X, U)\right)\right) \\
& \simeq \operatorname{Hom}_{R}(X, U),
\end{aligned}
$$

implying the first isomorphism. The other one is checked analogously.

Consider the triangular matrix extension

$$
T(R)=\left(\begin{array}{ll}
R & 0 \\
R & R
\end{array}\right)
$$

of $R$ and recall that $\mathcal{H}(R)$ is naturally equivalent to Mod $T(R)$, the category of left modules over $T(R)$. So let $F: \mathcal{H}(R) \longrightarrow$ Mod $T(R)$ denote this equivalence; we refer to [5] to see how $F$ acts.

Lemma 1.5. For every $\mathcal{X}$ in $\mathcal{H}(R),-\otimes_{\mathcal{H}} \mathcal{X} \simeq F(-) \otimes_{T(R)} F(\mathcal{X})$.

Proof. Notice first that, since $R$ is commutative, $T(R)^{\mathrm{op}}$ may be identified with $T(R)$ itself. Let $X=X \stackrel{f}{\rightarrow} Y$ and consider the composite functor

$$
G: \operatorname{Mod} T(R) \stackrel{F^{-1}}{\longrightarrow} \mathcal{H}(R) \stackrel{-\otimes_{\mathcal{H}} \mathcal{X}}{\longrightarrow} \operatorname{Mod} R
$$


Since $G$ is right exact and coproduct preserving, by [15, Theorem 3.33], $G \simeq-\otimes_{T(R)} B$ where $B=G(T(R))$ has a natural left $T(R)$-structure. Since $F^{-1}(T(R))=(0 \rightarrow R) \oplus$ $(R \rightarrow R)$, we get from Lemma 1.4 that

$$
B=\left((0 \rightarrow R) \otimes_{\mathcal{H}} \mathcal{X}\right) \oplus\left((R \rightarrow R) \otimes_{\mathcal{H}} \mathcal{X}\right) \simeq X \oplus Y .
$$

But, evidently, $X \oplus Y$ as a $T(R)$-module is nothing other than $F(\mathcal{X})$ itself; hence $G \simeq-\otimes_{T(R)} F(\mathcal{X})$ from which the required isomorphism of functors follows.

Lemma 1.5 tells us that one may use $-\otimes_{\mathcal{H}} \mathcal{X}$ freely to do homological algebra in the category $\mathcal{H}(R)$. For instance, with this notion of tensor product, one defines $\operatorname{Tor}_{i}^{\mathcal{H}}(\mathcal{X}, \mathcal{Y})$ for any two elements $\mathcal{X}, \mathcal{Y}$ in $\mathcal{H}(R)$ in the usual way: take a projective resolution for $\mathcal{X}$, apply $-\otimes_{\mathcal{H}} \mathcal{Y}$ and compute the homology modules. Note that it now routine to verify that, as in the case of modules over a commutative ring, Tor $_{i}^{\mathcal{H}}$ commutes with the usual localisation functor, that is, for any two objects $\mathcal{X}, \mathcal{Y}$ in $\mathcal{H}(R)$ and any prime ideal $p$ of $R$ there exists a natural isomorphism $\operatorname{Tor}_{i}^{\mathcal{H}}(\mathcal{X}, \mathcal{Y})_{\mathfrak{p}} \simeq$ $\operatorname{Tor}_{i}^{\mathcal{H}}\left(\mathcal{X}_{\mathrm{p}}, \mathcal{Y}_{\mathrm{p}}\right)$.

We point out that for any element $\mathcal{Y}$ in $\mathcal{H}(R)$, there exists a right exact functor $\mathcal{Y} \otimes_{\mathcal{H}}-: \mathcal{H}(R) \longrightarrow \operatorname{Mod} R$; this can be constructed directly from the functors $-\otimes_{\mathcal{H}} \mathcal{X}$ by using Yoneda's lemma [10, Ch. 5] or from Lemma 1.5 above. For future use, we record the following lemma whose proof is now standard in view of the notions of tensor product we already have.

Lemma 1.6. For any two elements $\mathcal{X}, \mathcal{Y}$ in $\mathcal{H}_{f g}(R)$, there exists an exact sequence $\mathcal{X}^{*} \otimes_{\mathcal{H}} \mathcal{Y} \longrightarrow \operatorname{Hom}_{\mathcal{H}}(\mathcal{X}, \mathcal{Y}) \longrightarrow \underline{\operatorname{Hom}}_{\mathcal{H}}(\mathcal{X}, \boldsymbol{Y}) \longrightarrow 0$

Definition 1.7. An object $X=X \stackrel{f}{\rightarrow} Y$ of $\mathcal{H}(R)$ is said to be locally projective on the punctured spectrum of $R$ provided $(X)_{\mathfrak{p}}=X_{\mathfrak{p}} \stackrel{f_{\mathfrak{p}}}{\rightarrow} Y_{\mathfrak{p}}$ is a projective object in $\mathcal{H}\left(R_{\mathfrak{p}}\right)$ for every nonmaximal prime ideal $\mathfrak{p}$ of $R$.

Assume now that $R$ is local Cohen-Macaulay with canonical module $\omega$. For an $R$-module $X, X^{\prime}$ denotes the $R$-module $\operatorname{Hom}_{R}(X, \omega)$, and if $X=X \stackrel{f}{\rightarrow} Y$ is an element in $\mathcal{H}(R)$, then $X^{\prime}$ will denote the object $\operatorname{Hom}_{R}(X, \omega)=\operatorname{Hom}_{R}(Y, \omega) \stackrel{\operatorname{Hom}_{R}(f, \omega)}{\longrightarrow} \operatorname{Hom}_{R}(X, \omega)$ of $\mathcal{H}(R)$.

With all these prerequisites in hand, we are now able to state the following theorem. As we pointed out earlier, its proof follows exactly that of [13, Proposition 12.16]; hence we skip it. Recall that for an object $\mathcal{X}$ in $\mathcal{H}(R)$, syz ${ }^{i}(\mathcal{X})$ is the $i$ th syzygy in a minimal projective presentation of $\mathcal{X}$. As usual, we ignore projective direct summands in $\operatorname{syz}^{i}(\mathcal{X})$. Also, $\mathrm{E}(R / \mathrm{m})$ stands for the injective envelope of $R / \mathrm{m}$.

THeorem 1.8. Let $R$ be a Cohen-Macaulay local ring of dimension d which possesses a canonical module $\omega$. Suppose that $\mathcal{X}$ is an object in $\mathcal{H}_{f g}(R)$ which is locally projective on the punctured spectrum of $R$ and $\mathcal{Y} \in \mathfrak{C}(\mathcal{H})$. Then there is an isomorphism 
of $\underline{\operatorname{End}}_{\mathcal{H}}(\mathcal{X})$-and $\underline{\operatorname{End}}_{\mathcal{H}}(\mathcal{Y})$-modules,

$$
\operatorname{Hom}_{R}\left(\underline{\operatorname{Hom}}_{\mathcal{H}}(\mathcal{X}, \mathcal{Y}), \mathrm{E}(R / \mathrm{m})\right) \simeq \operatorname{Ext}_{\mathcal{H}}^{1}\left(\mathcal{Y},\left(\operatorname{syz}^{d} \operatorname{Tr} \mathcal{X}\right)^{\prime}\right),
$$

which is natural in both variables.

Inspired by the above theorem, $\left(\operatorname{syz}^{d} \operatorname{Tr} \mathcal{X}\right)^{\prime}$ is called the Auslander-Reiten translate of $\mathcal{X}$ and is denoted by $\tau_{\mathcal{H}(R)}(\mathcal{X})$ or, when there is no ambiguity, $\tau(\mathcal{X})$.

\section{Proof of the main theorems}

Here, let $(R, m)$ be a complete Cohen-Macaulay local ring of Krull dimension $d$. In the following, we will prove the results mentioned in the introduction. As mentioned earlier, the proof of Theorem B proceeds by induction on $d$. More precisely, we shall reduce the problem by using regular elements. Details of basic properties of regular elements and how they behave with Cohen-Macaulay modules may be found in [6]. We just recall that for a finitely generated $R$-module $X$ a nonzero element $x \in R$ is said to be $X$-regular, or an $X$-sequence, provided it is not a zero divisor on $X$. Firstly, we define what we mean by a maximal Cohen-Macaulay object in $\mathcal{H}(R)$ and then collect some prerequisites. As before, $E$ is the injective envelope of $R / \mathrm{m}$.

Definition 2.1. An element $X=X \stackrel{f}{\rightarrow} Y$ of $\mathcal{H}_{f g}(R)$ is said to be (maximal) CohenMacaulay provided:

(i) $f$ is a monomorphism;

(ii) the $R$-modules $X, Y$ and $\operatorname{Coker}(f)$ are (maximal) Cohen-Macaulay.

Evidently, every maximal Cohen-Macaulay object lies in $\mathfrak{C}(\mathcal{H})$. Also, if $f$ is a monomorphism and either $X$ and $\operatorname{Coker}(f)$ or $Y$ and Coker $(f)$ are maximal CohenMacaulay, then $\mathcal{X}$ is maximal Cohen-Macaulay.

Definition 2.2. Let $\mathcal{X}=X \stackrel{f}{\rightarrow} Y$ belong to $\mathcal{H}_{f g}(R)$ and let $0 \neq x \in R$. We say that $x$ is an $X$-sequence provided it is both an $X$ - and a $\operatorname{Coker}(f)$-sequence.

For an object $\mathcal{X}=X \stackrel{f}{\rightarrow} Y$ of $\mathcal{H}_{f g}(R)$ and an element $0 \neq x \in R$, we denote by $\mathcal{X} / x \mathcal{X}$ the object $X \otimes_{R} R / x R \stackrel{f \otimes R / x R}{\longrightarrow} Y \otimes_{R} R / x R$ of $\mathcal{H}_{f g}(R / x R)$.

Lemma 2.3. If $\mathcal{X}=X \stackrel{f}{\rightarrow} Y$ is a (maximal) Cohen-Macaulay object of $\mathcal{H}(R)$ and $x \in R$ is an $\mathcal{X}$-sequence, then $\mathcal{X} / x \mathcal{X}$ is also (maximal) Cohen-Macaulay in $\mathcal{H}(R / x R)$.

Proof. By definition of (maximal) Cohen-Macaulay objects, the sequence $0 \longrightarrow$ $X \stackrel{f}{\longrightarrow} Y \stackrel{\pi_{f}}{\longrightarrow} \operatorname{Coker}(f) \longrightarrow 0$ is exact so that $x$ will also be a $Y$-sequence. It is well known that the $R / x R$-modules $X / x X, Y / x Y$ and $\operatorname{Coker}(f) / x \operatorname{Coker}(f)$ are then (maximal) Cohen-Macaulay; see [6, Theorem 2.1.3]. On the other hand, since $x$ is a Coker $(f)$-sequence, we have $\operatorname{Tor}_{1}^{R}(\operatorname{Coker}(f), R / x R)=0$ which in conjunction with the long exact sequence of Tor implies that $f \otimes R / x R$ is a monomorphism. This finishes the proof. 
Lemma 2.4. Let $\mathcal{X}$ be a locally projective object of $\mathcal{H}_{f g}(R)$ and let $x \in \mathrm{m}$ be an $R$ - and $\mathcal{X}$-sequence. If $\mathcal{L}$ is an arbitrary object of $\mathcal{H}_{f g}(R)$, then there exists a natural number $n$ such that $\underline{\operatorname{Hom}}_{\mathcal{H}(R)}(\mathcal{X}, \mathcal{L})$ is an $R / x^{n} R$-module and $\operatorname{Hom}_{R}\left(\underline{\operatorname{Hom}}_{\mathcal{H}(R)}(\mathcal{X}, \mathcal{L}), E\right) \simeq$ $\operatorname{Hom}_{R / x^{n} R}\left(\underline{\operatorname{Hom}}_{\mathcal{H}(R)}(X, \mathcal{L}), E^{\prime}\right)$ where $E^{\prime}$ is the injective envelope of

$$
\frac{R / x^{n} R}{\mathrm{~m} / x^{n} R}
$$

as an $R / x^{n} R$-module.

Proof. Since $\mathcal{X}$ is locally projective, the $R$-module $\underline{\operatorname{Hom}}_{\mathcal{H}}(\mathcal{X}, \mathcal{L})$ is of finite length. Hence there exists a natural number $n$ with $\mathrm{m}^{n} \underline{\operatorname{Hom}}_{\mathcal{H}(R)}(\mathcal{X}, \mathcal{L})=0$ and consequently $x^{n} \underline{\operatorname{Hom}}_{\mathcal{H}(R)}(\mathcal{X}, \mathcal{L})=0$. So $\underline{\operatorname{Hom}}_{\mathcal{H}(R)}(\mathcal{X}, \mathcal{L})$ is an $R / x^{n} R$-module. Now, by using the Hom $-\otimes$ adjoint isomorphism,

$$
\begin{aligned}
\operatorname{Hom}_{R}\left(\underline{\operatorname{Hom}}_{\mathcal{H}(R)}(X, \mathcal{L}), E\right) & \simeq \operatorname{Hom}_{R}\left(\frac{R}{x^{n} R} \otimes_{R / x^{n} R} \underline{\operatorname{Hom}}_{\mathcal{H}(R)}(X, \mathcal{L}), E\right) \\
& \simeq \operatorname{Hom}_{R / x^{n} R}\left(\underline{\operatorname{Hom}}_{\mathcal{H}(R)}(\mathcal{X}, \mathcal{L}), \operatorname{Hom}_{R}\left(R / x^{n} R, E\right)\right) .
\end{aligned}
$$

Since $\operatorname{Hom}_{R}\left(R / x^{n} R, E\right)=E^{\prime}$, the required isomorphism follows.

Following [7], If $\mathcal{X}$ is an element in $\mathcal{H}_{f g}(R)$ and $x \in \mathrm{m}$ is both an $R$ - and $\mathcal{X}$-sequence, we let $n_{x}(\mathcal{X})$ be the minimum of all $n$ such that $x^{n} \operatorname{Ext}_{\mathcal{H}}^{1}(\mathcal{X},-)=0$.

Lemma 2.5. Let $\mathcal{X}$ be a nonprojective locally projective object of $\mathfrak{S}(\mathcal{H})$ and let $x \in \mathrm{m}$ be an $R$-and $\mathcal{X}$-sequence. Assume that $\mathcal{L}$ is an object of $\mathfrak{C}(\mathcal{H})$ over which $x$ is a regular sequence. Then, for some natural number $n$, there exists a natural map

$$
\eta_{\mathcal{X}, \mathcal{L}}: \operatorname{Hom}_{R / x^{n} R}\left(\underline{\operatorname{Hom}}_{\mathcal{H}\left(R / x^{n} R\right)}\left(\mathcal{X} / x^{n} \mathcal{X}, \mathcal{L} / x^{n} \mathcal{L}\right), E^{\prime}\right) \longrightarrow \operatorname{Hom}_{R}\left(\underline{\operatorname{Hom}}_{\mathcal{H}(R)}(\mathcal{X}, \mathcal{L}), E\right)
$$

Moreover, if $n_{x}(\mathcal{L})$ is finite and there exists a map $\mathcal{X} \rightarrow \mathcal{L}$ which does not factor through projective objects then, for some natural number $n$, there exists a nonzero $\eta_{\mathcal{X}, \mathcal{L}}$ as above.

Proof. According to Lemma 2.4, we pick a natural number $n_{1}$ such that the $R$-module $\underline{\operatorname{Hom}}_{\mathcal{H}(R)}(\mathcal{X}, \mathcal{L})$ is an $R / x^{n_{1}} R$-module and $\operatorname{Hom}_{R}\left(\underline{\operatorname{Hom}}_{\mathcal{H}(R)}(\mathcal{X}, \mathcal{L}), E\right)$ is isomorphic to $\operatorname{Hom}_{R / x^{n}{ }_{1} R}\left(\underline{\operatorname{Hom}}_{\mathcal{H}(R)}(\mathcal{X}, \mathcal{L}), E^{\prime}\right)$. Note that the natural map

$$
\operatorname{Hom}_{\mathcal{H}(R)}(\mathcal{X}, \mathcal{L}) \longrightarrow \operatorname{Hom}_{\mathcal{H}\left(R / x^{n_{1}} R\right)}\left(\mathcal{X} / x^{n_{1}} \mathcal{X}, \mathcal{L} / x^{n_{1}} \mathcal{L}\right)
$$

induces a map

$$
\varphi_{X, \mathcal{L}}: \underline{\operatorname{Hom}}_{\mathcal{H}(R)}(\mathcal{X}, \mathcal{L}) \longrightarrow \underline{\operatorname{Hom}}_{\mathcal{H}\left(R / x^{n_{1}} R\right)}\left(\mathcal{X} / x^{n_{1}} \mathcal{X}, \mathcal{L} / x^{n_{1}} \mathcal{L}\right)
$$

because for any projective $R$-module $Q, Q / x^{n_{1}} Q$ is a projective $R / x^{n_{1}} R$-module by [6, Lemma 1.3.5]. Hence the required map follows as $\eta_{X, \mathcal{L}}=\operatorname{Hom}_{R / x^{n}{ }_{R}}\left(\varphi_{X, \mathcal{L}}, E^{\prime}\right)$.

For the second part let the sequence $0 \longrightarrow \mathcal{K} \stackrel{i}{\longrightarrow} \mathcal{P} \stackrel{j}{\longrightarrow} \mathcal{L} \longrightarrow 0$ be exact with $\mathcal{P}$ the projective cover. We know from the assumption that $n_{x}(\mathcal{X})$ is finite; 
also $n_{x}(\mathcal{K})$ is by [7, Proposition 1.7] finite. Set $n \geq \max \left\{n_{1}, n_{x}(\mathcal{X}), n_{x}(\mathcal{L})\right\}+1$. We will then show that the map $\varphi_{X, \mathcal{L}}$ which arises from the above process with $n$ instead of $n_{1}$ is a monomorphism. Suppose that $\epsilon: \mathcal{X} \longrightarrow \mathcal{L}$ is such that $\epsilon \otimes_{R} R / x^{n} R: \mathcal{X} / x^{n} \mathcal{X} \longrightarrow \mathcal{L} / x^{n} \mathcal{L}$ factors through a projective object in $\mathcal{H}\left(R / x^{n} R\right)$. We will show that $\epsilon$ factors through a projective object in $\mathcal{H}(R)$. By [12] it suffices to show that in the pullback diagram

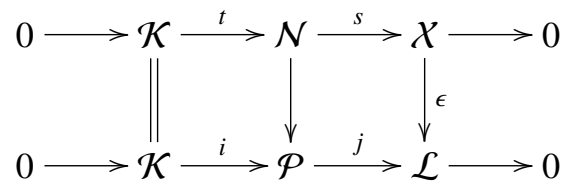

the top row splits. Since $x$ is an $\mathcal{L}$ - and $\mathcal{X}$-sequence, we get the diagram

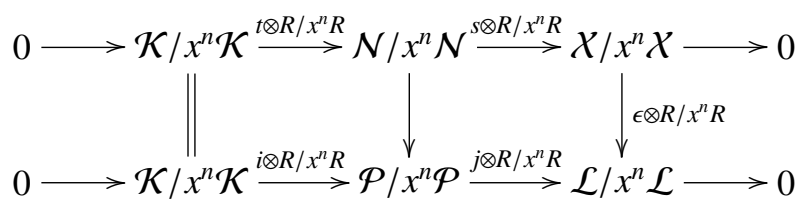

Since $\epsilon \otimes R / x^{n} R$ factors through a projective object, the top row of the latter diagram splits, that is, $\mathcal{N} / x^{n} \mathcal{N} \simeq \mathcal{K} / x^{n} \mathcal{K} \oplus \mathcal{X} / x^{n} \mathcal{X}$ or $\mathcal{N} / x^{n} \mathcal{N} \simeq(\mathcal{X} \oplus \mathcal{K}) / x^{n}(\mathcal{X} \oplus \mathcal{K})$. Since $n \geq n_{x}(\mathcal{X} \oplus \mathcal{K})+1, \mathcal{X} \oplus \mathcal{K} \in \mathbb{C}(\mathcal{H})$, and $x$ is an $\mathcal{N}$-sequence, [7, Theorem 1.3] yields that $\mathcal{N} \simeq X \oplus \mathcal{K}$. But then, by [14], we deduce that the sequence $0 \longrightarrow \mathcal{K} \stackrel{t}{\longrightarrow} \mathcal{N} \stackrel{s}{\longrightarrow}$ $\mathcal{X} \longrightarrow 0$ is split, as required. Consequently $\varphi_{\mathcal{X}, \mathcal{L}}$ is a monomorphism which, by virtue of the existence of a map which does not factor through projective objects, is certainly nonzero; thus, so is $\eta_{X, \mathcal{L}}$.

Combining the above lemma and Theorem 1.8, we obtain the following corollary.

COROLlaRy 2.6. Under the assumptions of Lemma 2.5 there exists, for some natural number $n$, a natural map

$$
\operatorname{Ext}_{\mathcal{H}\left(R / x^{n} R\right)}^{1}\left(\mathcal{L} / x^{n} \mathcal{L}, \tau_{\mathcal{H}\left(R / x^{n} R\right)}\left(\mathcal{X} / x^{n} \mathcal{X}\right)\right) \rightarrow \operatorname{Ext}_{\mathcal{H}(R)}^{1}\left(\mathcal{L}, \tau_{\mathcal{H}(R)}(\mathcal{X})\right)
$$

still denoted by $\eta_{X, \mathcal{L}}$. If the extra assumptions of Lemma 2.5 hold, $\eta_{X, \mathcal{L}}$ is nonzero.

Proposition 2.7. Let $\mathcal{X}$ be a nonprojective locally projective object of $\mathfrak{C}(\mathcal{H})$ with local endomorphism ring. Assume that $x \in \mathrm{m}$ is an $R$ - and $\mathcal{X}$-sequence. Then for some natural number $n$, there exists a nonzero natural map

$$
\eta_{\mathcal{X}}: \operatorname{Hom}_{R / x^{n} R}\left(\underline{\operatorname{End}}_{\mathcal{H}\left(R / x^{n} R\right)}\left(\mathcal{X} / x^{n} \mathcal{X}\right), E^{\prime}\right) \longrightarrow \operatorname{Hom}_{R}\left(\underline{\operatorname{End}}_{\mathcal{H}(R)}(\mathcal{X}), E\right)
$$

with the property that every socle element of the right-hand side is the image of a socle element of the left-hand side. 
Proof. Set $\Gamma=\underline{\operatorname{End}}_{\mathcal{H}(R)}(\mathcal{X})$ and consider the natural number $n \geq n_{x}(\mathcal{X})+1$ of Lemma 2.5 which corresponds to a nonzero map $\varphi_{\mathcal{X}}: \Gamma \longrightarrow \Gamma^{\prime}$ where $\Gamma^{\prime}=$ $\underline{\operatorname{End}}_{\mathcal{H}\left(R / x^{n} R\right)}\left(\mathcal{X} / x^{n} \mathcal{X}\right)$. This gives the asserted nonzero map $\eta_{\mathcal{X}}=\operatorname{Hom}_{R / x^{n} R}\left(\varphi_{\mathcal{X}}, E^{\prime}\right)$. By [7, Theorem 1.3], $\Gamma^{\prime}$ is also a local ring, and by a result from Auslander [1, Proposition 2.5], $\mathfrak{P}(\mathcal{X}, \mathcal{X}) \subseteq J(\operatorname{End}(\mathcal{X}))$ and $\mathfrak{P}\left(\mathcal{X} / x^{n} \mathcal{X}, \mathcal{X} / x^{n} \mathcal{X}\right) \subseteq J\left(\operatorname{End}\left(\mathcal{X} / x^{n} \mathcal{X}\right)\right)$ where $J$ denotes the Jacobson radical. Hence $J(\Gamma)=J(\operatorname{End}(\mathcal{X})) / \mathfrak{P}(\mathcal{X}, \mathcal{X})$ and, likewise, $J\left(\Gamma^{\prime}\right)=J\left(\operatorname{End}\left(\mathcal{X} / x^{n} \mathcal{X}\right)\right) / \mathfrak{P}\left(\mathcal{X} / x^{n} \mathcal{X}, \mathcal{X} / x^{n} \mathcal{X}\right)$. Note that if an endomorphism $\iota$ of $\mathcal{X}$ factors through a projective object, then so does the endomorphism $\iota \otimes R / x^{n} R$ of $\mathcal{X} / x^{n} \mathcal{X}$. Therefore one may apply [6, Lemma 3.3.2] to deduce that $\varphi_{\mathcal{X}}$ induces a map $J(\Gamma) \longrightarrow J\left(\Gamma^{\prime}\right)$. So now there is a ring homomorphism $\psi_{x}: \Gamma / J(\Gamma) \longrightarrow \Gamma^{\prime} / J\left(\Gamma^{\prime}\right)$ which is nonzero because otherwise the unit element of $\Gamma^{\prime}$ would lie in $J\left(\Gamma^{\prime}\right)$ which is absurd. Moreover, $\psi_{\mathcal{X}}$ is mono since $\Gamma / J(\Gamma)$ is simple. Now consider the commutative diagram

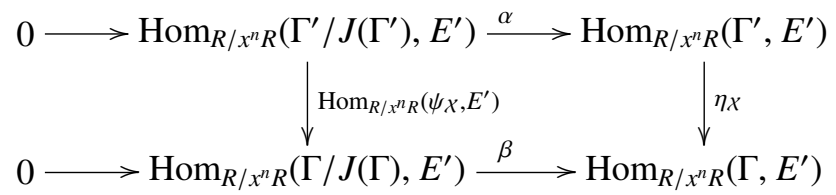

in which $\operatorname{Hom}_{R / x^{n} R}\left(\psi_{\chi}, E^{\prime}\right)$ is an epimorphism and $\alpha$ and $\beta$ are just the obvious maps. Suppose that $h \in \operatorname{Hom}_{R / x^{n} R}\left(\Gamma, E^{\prime}\right)$ is a socle element. Then there exists an element $h^{\prime} \in \operatorname{Hom}_{R / x^{n} R}\left(\Gamma / J(\Gamma), E^{\prime}\right)$ with $h=\beta\left(h^{\prime}\right)$. Since $h^{\prime}$ itself is the image of some element $h^{\prime \prime} \in \operatorname{Hom}_{R / x^{n} R}\left(\Gamma^{\prime} / J\left(\Gamma^{\prime}\right), E^{\prime}\right)$, we get $h=\beta \circ \operatorname{Hom}_{R / x^{n} R}\left(\psi_{X}, E^{\prime}\right)\left(h^{\prime \prime}\right)=\eta_{X} \circ \alpha\left(h^{\prime \prime}\right)$. But, clearly, $J\left(\Gamma^{\prime}\right)$ annihilates $\alpha\left(h^{\prime \prime}\right)$, that is, $\alpha\left(h^{\prime \prime}\right)$ is a socle element. This finishes the proof.

The following theorem is a combination of the above proposition with Theorem 1.8.

Theorem 2.8. Under the assumptions of Proposition 2.7, for some natural number $n$, there exists a nonzero natural map

$$
\eta_{\mathcal{X}}: \operatorname{Ext}_{\mathcal{H}\left(R / x^{n} R\right)}^{1}\left(\mathcal{X} / x^{n} \mathcal{X}, \tau_{\mathcal{H}\left(R / x^{n} R\right)}\left(\mathcal{X} / x^{n} \mathcal{X}\right)\right) \longrightarrow \operatorname{Ext}_{\mathcal{H}(R)}^{1}\left(\mathcal{X}, \tau_{\mathcal{H}(R)}(\mathcal{X})\right)
$$

which has the property that every socle element of the right-hand side is the image of a socle element of the left-hand side.

The following proposition is the last thing we need to prove the existence of almost split sequences.

Proposition 2.9. Let $\mathcal{X}$ be a locally projective Cohen-Macaulay object of $\mathcal{H}(R)$ with a local endomorphism ring. Then $\tau_{\mathcal{H}(R)}(\mathcal{X})=\left(\operatorname{syz}^{d} \operatorname{Tr} \mathcal{X}\right)^{\prime}$ has a local endomorphism ring.

Proof. From the assumption, we get that $\underline{\operatorname{End}}_{\mathcal{H}(R)}(\operatorname{Tr} \mathcal{X})$ is local. For every projective object $\mathcal{P}$ in $\mathcal{H}_{f g}(R), \operatorname{Ext}_{\mathcal{H}(R)}^{1}(\operatorname{Tr} \mathcal{X}, \mathcal{P})=0$ by Proposition 1.2 ; hence one applies a standard argument to show that $\underline{\operatorname{End}}_{\mathcal{H}(R)}(\operatorname{Tr} \mathcal{X}) \simeq \underline{\operatorname{End}}_{\mathcal{H}(R)}\left(\operatorname{syz}^{1} \operatorname{Tr} \mathcal{X}\right)$ implying that the latter is a local ring. Using this repeatedly, it follows that $\underline{\operatorname{End}}_{\mathcal{H}(R)}\left(\operatorname{syz}^{d} \operatorname{Tr} \mathcal{X}\right)$ is 
also local. But syz ${ }^{d} \operatorname{Tr} \mathcal{X}$ has no projective direct summands. Therefore $\mathfrak{P}\left(\operatorname{syz}^{d} \operatorname{Tr} \mathcal{X}\right.$, $\left.\operatorname{syz}^{d} \operatorname{Tr} \mathcal{X}\right)$ is contained in $J\left(\operatorname{End}\left(\operatorname{syz}^{d} \operatorname{Tr} \mathcal{X}\right)\right)$ by [1, Proposition 2.5]. This gives that $\operatorname{End}_{\mathcal{H}(R)}\left(\operatorname{syz}^{d} \operatorname{Tr} \mathcal{X}\right)$ is a local ring. Note that $\operatorname{syz}^{d} \operatorname{Tr} \mathcal{X}$ is a Cohen-Macaulay element in $\mathcal{H}(R)$. Consequently, since $(-)^{\prime}$ is clearly a duality on the subcategory of CohenMacaulay objects, we elicit the desired result.

We now come to the proof of Theorem B.

Proof of Theorem B. Since $\mathcal{X}$ is locally projective, $\operatorname{Hom}_{R}\left(\underline{\operatorname{End}}_{\mathcal{H}(R)}(\mathcal{X}), E\right)$ has a socle element, that is, an element which vanishes over $J\left(\underline{\operatorname{End}}_{\mathcal{H}(R)}(\mathcal{X})\right)$. So by Theorem 1.8, $\operatorname{Ext}_{\mathcal{H}(R)}^{1}\left(\mathcal{X}, \tau_{\mathcal{H}(R)}(\mathcal{X})\right)$ has a socle element $s: 0 \longrightarrow \tau_{\mathcal{H}(R)}(\mathcal{X}) \stackrel{\delta}{\longrightarrow} \mathcal{E} \stackrel{\theta}{\longrightarrow} \mathcal{X} \longrightarrow 0$. Our aim is to prove, by induction on $d$, that this element is indeed the almost split sequence ending in $\mathcal{X}$ in the subcategory $\mathfrak{C}(\mathcal{H})$. That $\tau_{\mathcal{H}(R)}(\mathcal{X})$, and so $\mathcal{E}$, lie in $\mathfrak{C}(\mathcal{H})$ follows from Proposition 1.2. By virtue of Proposition 2.9, we only need to show that $\theta$ is a right almost split map. For let $\mathcal{L} \stackrel{\epsilon}{\longrightarrow} X$ be a nonisomorphism with $\mathcal{L}$ an indecomposable object in $\mathfrak{S}(\mathcal{H})$. Then there exists a pullback diagram

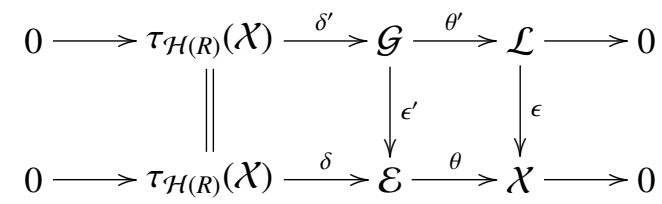

Clearly, it is sufficient to show that the sequence $t: 0 \rightarrow \tau_{\mathcal{H}(R)}(\mathcal{X}) \stackrel{\delta^{\prime}}{\rightarrow} \mathcal{G} \stackrel{\theta^{\prime}}{\rightarrow} \mathcal{L} \rightarrow 0$ is split. Since $\mathcal{X}$ and $\mathcal{L}$ are maximal Cohen-Macaulay as $R$-modules, there exists an element $x \in \mathfrak{m}$ which is simultaneously an $R$-, $\mathcal{X}$-, and $\mathcal{L}$-sequence. Assume first that $n_{x}(\mathcal{L})$ is finite. Then by Theorem 2.8 and Corollary 2.6 we may choose a natural number $n \geq \max \left\{n_{x}(\mathcal{X}), n_{x}(\mathcal{L})\right\}+1$ in such a way that there exists a nonzero map

$$
\eta_{\mathcal{X}}: \operatorname{Ext}_{\mathcal{H}\left(R / x^{n} R\right)}^{1}\left(\mathcal{X} / x^{n} \mathcal{X}, \tau_{\mathcal{H}\left(R / x^{n} R\right)}\left(\mathcal{X} / x^{n} \mathcal{X}\right)\right) \longrightarrow \operatorname{Ext}_{\mathcal{H}(R)}^{1}\left(\mathcal{X}, \tau_{\mathcal{H}(R)}(\mathcal{X})\right)
$$

and also a map

$$
\eta_{X, \mathcal{L}}: \operatorname{Ext}_{\mathcal{H}\left(R / x^{n} R\right)}^{1}\left(\mathcal{L} / x^{n} \mathcal{L}, \tau_{\mathcal{H}\left(R / x^{n} R\right)}\left(\mathcal{X} / x^{n} \mathcal{X}\right)\right) \longrightarrow \operatorname{Ext}_{\mathcal{H}(R)}^{1}\left(\mathcal{L}, \tau_{\mathcal{H}(R)}(\mathcal{X})\right)
$$

and such that, by [7, Theorem 1.3], $\mathcal{X} / x^{n} \mathcal{X}$ and $\mathcal{L} / x^{n} \mathcal{L}$ both have local endomorphism rings and, moreover, $s$ is the image under $\eta_{\mathcal{X}}$ of a socle element

$$
s^{\prime}: 0 \longrightarrow \tau_{\mathcal{H}\left(R / x^{n} R\right)}\left(\mathcal{X} / x^{n} \mathcal{X}\right) \stackrel{\sigma}{\longrightarrow} \mathcal{E}^{\prime} \stackrel{v}{\longrightarrow} \mathcal{X} / x^{n} \mathcal{X} \longrightarrow 0
$$

of $\operatorname{Ext}_{\mathcal{H}\left(R / x^{n} R\right)}^{1}\left(\mathcal{X} / x^{n} \mathcal{X}, \tau_{\mathcal{H}\left(R / x^{n} R\right)}\left(\mathcal{X} / x^{n} \mathcal{X}\right)\right)$. By the induction hypothesis $s^{\prime}$ is an almost split sequence. Now consider the pullback diagram

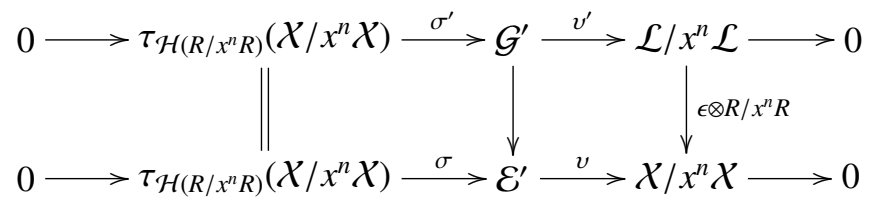


in which the map $\epsilon \otimes R / x^{n} R$ is not an isomorphism by [6, Lemma 3.3.2]. Therefore the sequence $t^{\prime}: 0 \longrightarrow \tau_{\mathcal{H}\left(R / x^{n} R\right)}\left(\mathcal{X} / x^{n} \mathcal{X}\right) \stackrel{\sigma^{\prime}}{\longrightarrow} \mathcal{G}^{\prime} \stackrel{v^{\prime}}{\longrightarrow} \mathcal{L} / x^{n} \mathcal{L} \longrightarrow 0$ must be split exact. But then the commutative diagram

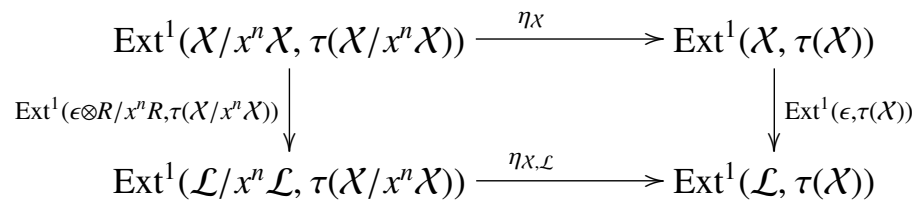

implies that $t=0$ as desired.

Suppose now that $n_{x}(\mathcal{L})$ is infinite. Still, for some natural number $n \geq n_{x}(\mathcal{X})+1$, we have the map $\eta_{\mathcal{X}, \mathcal{L}}$ and also the nonzero map $\eta_{\mathcal{X}}$ with the aforementioned property. We claim that $\epsilon \otimes R / x^{n} R$ is not a split epimorphism. For if this is the case then Nakayama's lemma gives that $\epsilon$ is an epimorphism. Our choice of $n$ shows that $x^{n} \operatorname{Ext}^{1}(X,-)=0$, that is, the map $x^{n}: \mathcal{X} \rightarrow \mathcal{X}$ given by multiplication by $x^{n}$ factors through a projective object by [12]; hence so does the map $x^{n} \epsilon$. On the other hand, the exact sequence of Lemma 1.6 gives rise to the commutative diagram

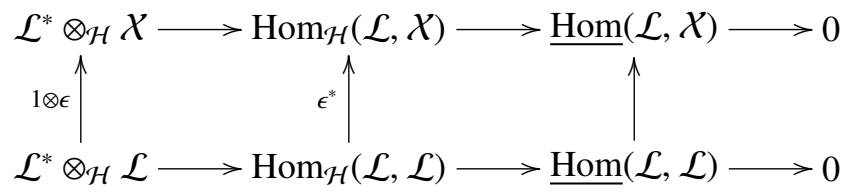

in which the map $1 \otimes \epsilon$ is an epimorphism since $\mathcal{L}^{*} \otimes_{\mathcal{H}}-$ is a right exact functor. Note that the image of the horizontal maps in the left square coincide with $\mathfrak{P}(\mathcal{L}, \mathcal{X})$ and $\mathfrak{P}(\mathcal{L}, \mathcal{L})$, respectively. Therefore, chasing this diagram, one obtains a map $\gamma: \mathcal{L} \rightarrow \mathcal{L}$ which factors through a projective object and satisfies $x^{n} \epsilon=\epsilon \gamma$. So if $x^{n}: \mathcal{L} \rightarrow \mathcal{L}$ is multiplication by $x^{n}$, then $\epsilon\left(\gamma-x^{n}\right)=0$. Now the entry $\mathcal{Y}$ in the pullback diagram

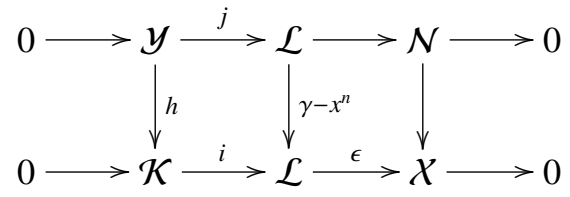

is, by definition of a pullback, a submodule of $\mathcal{K} \oplus \mathcal{L}$ consisting of all pairs $((\gamma-$ $\left.x^{n}\right)(\ell), \ell$ ) with $\ell \in \mathcal{L}$. (We are looking at modules over some triangular matrix ring instead of objects in $\mathcal{H}(R)$ as pointed out before.) This means that $\mathcal{Y} \simeq \operatorname{Im}\left(\gamma-x^{n}\right) \oplus \mathcal{L}$ and that the map $j$ is an isomorphism. Hence $\mathcal{L} \simeq \mathcal{L} \oplus \operatorname{Im}\left(\gamma-x^{n}\right)$ which implies that $\gamma=x^{n}$ since $\mathcal{L}$ is indecomposable. This conflicts with $n_{x}(\mathcal{L})=\infty$. Hence $\epsilon \otimes R / x^{n} R$ is not a split epimorphism so that it factors through $v$. The rest of the proof goes as in the first case and gives $t=0$.

Proof of Theorem C. For a maximal Cohen-Macaulay object $X=X \stackrel{f}{\rightarrow} Y$ of $\mathcal{H}(R)$ with $\operatorname{Ext}_{\mathcal{H}}^{1}(\operatorname{Coker}(f), R)=0, \operatorname{Tr}_{\mathcal{H}}(\mathcal{X})$ is by Proposition 1.2 a representative of the 
induced monomorphism $\operatorname{Tr} \operatorname{Coker}(f) \stackrel{\operatorname{Tr}\left(\pi_{f}\right)}{\longrightarrow} \operatorname{Tr} Y$. Moreover by [11, Lemma 2.2] and the construction of projective covers in $\mathcal{H}(R)$ given in Lemma $1.1, \operatorname{syz}^{d} \operatorname{Tr} \mathcal{X}$ may be represented as syz ${ }^{d} \operatorname{Tr} \operatorname{Coker}(f) \rightarrow \operatorname{syz}^{d} \operatorname{Tr} Y$. Now apply the functor $(-)^{\prime}$ as defined before.

\section{References}

[1] M. Auslander, 'Comments on the functor Ext', Topology 8 (1969), 151-166.

[2] M. Auslander, 'Functors and morphisms determined by objects', in: Proc. Conf. on Representation Theory (Philadelphia 1967) (Dekker, New York, 1978), 1-244.

[3] M. Auslander and I. Reiten, 'Representation theory of Artin algebras III', Comm. Algebra 3 (1975), 239-294.

[4] M. Auslander and I. Reiten, 'Almost split sequences for Cohen-Macaulay modules', Math. Ann. 277(2) (1987), 345-349.

[5] M. Auslander, I. Reiten and S. O. Smalø, Representation Theory of Artin Algebras, Cambridge Studies in Advanced Mathematics, 36 (Cambridge University Press, Cambridge, 1995).

[6] W. Bruns and J. Herzog, Cohen-Macaulay Rings, Cambridge Studies in Advanced Mathematics, 39 (Cambridge University Press, Cambridge, 1993).

[7] S. Ding and Ø. Solberg, 'The Maranda theorem and liftings of modules', Comm. Algebra 21(4) (1993), 1161-1187.

[8] E. Enochs and S. Estrada, 'Projective representations of quivers', Comm. Algebra 33 (2005), 3467-3478.

[9] E. Enochs, S. Estrada and J. R. García Rozas, 'Injective representations of infinite quivers. Applications', Canad. J. Math. 61(2) (2009), 315-335.

[10] P. Freyd, Abelian Categories (Harper \& Row, New York, 1964).

[11] Y. Guo, 'The class of modules with projective cover', J. Korean Math. Soc. 46(1) (2009), 51-58.

[12] P. Hilton and D. Rees, 'Natural maps of extension functors and a theorem of R. G. Swan', Proc. Camb. Phil. Soc. Math. Phys. Sci. 51 (1961).

[13] G. Leuschke and R. Wiegand, Cohen-Macaulay Representations (American Mathematical Society, Providence, RI, 2012).

[14] T. Miyata, 'Note on direct summands of modules', J. Math. Kyoto Univ. 7(1) (1967), 65-69.

[15] J. J. Rotman, An Introduction to Homological Algebra (Academic Press, New York, 1979).

[16] Sh. Salarian and R. Vahed, Almost split sequences for complexes of maximal Cohen-Macaulay modules, in preparation.

[17] Y. Yoshino, Cohen-Macaulay Modules over Cohen-Macaulay Rings, London Mathematical Society Lecture Note Series, 146 (Cambridge University Press, Cambridge, 1990).

HOSSEIN ESHRAGHI, Department of Pure Mathematics,

Faculty of Mathematical Sciences, University of Kashan,

PO Box 87317-51167, Kashan, Iran

e-mail: eshraghi@kashanu.ac.ir 